


\title{
Plasma Ang2 and ADAM17 levels are elevated during clinical malaria; Ang2 level correlates with severity \\ and expression of EPCR-binding PfEMP1
}

Received: 12 July 2016

Accepted: 07 October 2016

Published: 27 October 2016
Jens E. V. Petersen ${ }^{1, *}$, Sixbert I. Mkumbaye ${ }^{2, *}{ }^{*}$ Anna V. Vaaben ${ }^{1}$, Alphaxard Manjurano ${ }^{3}$, Eric Lyimo ${ }^{3}$, Reginald A. Kavishe ${ }^{2}$, Steven B. Mwakalinga ${ }^{2}$, Jacklin Mosha ${ }^{3}$, Daniel T. R. Minja ${ }^{4}$, John P. A. Lusingu ${ }^{4}$, Thor G. Theander ${ }^{1}$, Thomas Lavstsen ${ }^{1}$ \& Christian W. Wang ${ }^{1}$

The pathogenesis of Plasmodium falciparum malaria involves a complex interplay between parasite adhesion and inflammatory response that includes release of cytokines and activation of the endothelium with accompanying release of Angiopoitin 2 (Ang2) to the plasma. A-disintegrin and metalloproteinase 17 (ADAM17) is a protein responsible for releasing cytokines, including Tumor Necrosis Factor $\alpha$ (TNF $\alpha$ ), and shedding of adhesion proteins. In this study, we show that plasma levels of ADAM17 are increased in Tanzanian children hospitalized with a malaria infection compared with asymptomatic children but similar to children hospitalized with other infectious diseases. The plasma levels of ADAM17 decreased during recovery after an acute malaria episode. Plasma levels of Ang2 were associated with markers of malaria severity and levels of var transcripts encoding $P$. falciparum Erythrocyte Membrane Protein 1 (PfEMP1) containing Cysteine Rich Inter Domain Region $\alpha 1$ (CIDRo1) domains predicted to bind Endothelial Protein C receptor (EPCR). ADAM17 levels were not associated with expression of var genes encoding different PfEMP1 types when controlling for age. These data are the first to report ADAM17 plasma levels in malaria-exposed individuals, and support the notion that parasite sequestration mediated by EPCR-binding PFEMP1 is associated with endothelial activation and pathology in severe paediatric malaria.

In 2015, there were between 150 and 300 million malaria cases worldwide, causing an estimated 438,000 deaths of which $90 \%$ occurred in Sub-Saharan Africa ${ }^{1}$. The most vulnerable group is children under the age of five infected with Plasmodium falciparum. Even with the current artemisinin combination treatments, infected individuals are at a high risk of dying if they develop symptoms or signs of severe malaria (SM). Clinical manifestations of SM are typically cerebral malaria (CM), respiratory distress (RD), severe malarial anaemia (SMA), and hyperparasitaemia and they often overlap in individual patients. A hallmark of a $P$. falciparum infection is the sequestration of infected erythrocytes (IEs) to the host microvasculature ${ }^{2}$. To avoid splenic clearance, the parasite adheres to the vasculature by expressing P. falciparum Erythrocyte Membrane Protein 1 (PfEMP1) binding infected erthrocytes to endothelial host receptors such as cluster of differentiation 36 (CD36) and endothelial protein C receptor $(\mathrm{EPCR})^{3}$.

PfEMP1s are encoded by var genes, and each $P$. falciparum genome contains about 60 different var genes that are divided into four main groups: A, B, C, and var2csa, correlating both with genomic location and direction of transcription, sequence similarity and receptor binding phenotype ${ }^{3-6}$. Thus, group A PfEMP1s, characterized

${ }^{1}$ Centre for Medical Parasitology, Department of Immunology \& Microbiology, Faculty of Health and Medical Sciences, University of Copenhagen and Department of Infectious Diseases, Rigshospitalet, Copenhagen, Denmark. ${ }^{2}$ Kilimanjaro Christian Medical University College and Kilimanjaro Clinical Research Institute, Moshi, Tanzania. ${ }^{3}$ National Institute for Medical Research, Mwanza Research Centre, Mwanza, Tanzania. ${ }^{4}$ National Institute for Medical Research, Tanga Centre, Tanga, Tanzania. ${ }^{*}$ These authors contributed equally to this work. Correspondence and requests for materials should be addressed to J.E.V.P. (email: Jevang@sund.ku.dk) 
by having an N-terminal Duffy-binding like $\alpha 1$ (DBL $\alpha 1)$ domains followed by either a CIDR $\alpha 1$ domain, which is predicted to bind EPCR or a $\operatorname{CIDR} \beta, \gamma$, or $\delta$ domain, which are potentially linked to rosetting (IE binding of uninfected erythrocytes). Group B and C PfEMP1s have N-terminal DBL $\alpha 0$-CIDR $\alpha 2-6$ domain cassettes (co-occurring domain subtypes) predicted to bind CD36. A special set of PfEMP1s, the domain cassette 8 (DC8) PfEMP1, have evolved as an intermediate of group B and A PfEMP1. The DC8 PfEMP1 also bind EPCR via the CIDR $\alpha 1.1$ or 1.8 domain subtypes. Parasite sequestration is a major driver of the pathogenesis of an P. falciparum infection ${ }^{2}$, and sequestration via EPCR-binding PfEMP1s has been strongly associated with the development of $\mathrm{SM}^{3,7-9}$.

P. falciparum infections activate the innate immune system causing release of inflammatory cytokines. Of particular importance is Tumor Necrosis Factor $\alpha(\mathrm{TNF} \alpha)$, a proinflammatory cytokine that induces fever, cachexia, and apoptosis of endothelial cells ${ }^{10,11}$. TNF $\alpha$ is increased in malaria patients and has been associated with the severity of infection ${ }^{12}$. TNFo is released by A-disintegrin and metalloproteinase 17 (ADAM17), primarily from macrophages where it is expressed on the surface and subsequently cleaved off ${ }^{13}$. ADAM17 was initially discovered as the metalloproteinase responsible for cleaving and releasing TNF $\alpha$ and is therefore also known as TNF $\alpha$ converting enzyme (TACE) $)^{13}$.

ADAM17 mediates shedding of multiple other cytokines and cytokine receptors, including colony stimulating factor $1(\mathrm{CSF} 1)$, TNF $\alpha$ receptor $(\mathrm{TNF} \alpha \mathrm{R})$ and interleukin 6 receptor (IL6R) ${ }^{14}$. ADAM17 also mediates shedding of proteins involved in leukocyte-endothelial cell interactions, such as L-selectin and intercellular adhesion molecule-1 (ICAM-1) ${ }^{14,15}$, as well as a range of other surface receptors including EPCR ${ }^{14,16}$. Thus, ADAM17 may influence availability of host receptors utilized by IE in malaria. Absence of EPCR-staining at sites of the brain with sequestering IEs suggests that EPCR is cleaved off the endothelium or internalized as a consequence of sequestration, potentially exacerbating the vascular pathology by impairing the functions of activated Protein C $(\mathrm{APC})^{17}$.

The interaction between EPCR and PfEMP1 is also thought to enhance the vascular pathology, as PfEMP1 binding to EPCR blocks the binding of the natural EPCR ligand, Protein C (PC), in vitro ${ }^{6,18}$. In vitro, PfEMP1 binding to EPCR reduces PC activation and dampens APC's anti-inflammatory effects, including the ability to protect against thrombin-induced barrier disruption ${ }^{19,20}$. In the absence of APC-EPCR engagement, thrombin cleaving of protease-activated receptor 1 (PAR1) results in enhanced signaling for release of Weibel-Palade Bodies (WPBs).

Angiopoietin 2 (Ang2) is a growth factor molecule released from $\mathrm{WPBs}^{21}$. Elevated plasma concentrations of Ang2 reflect endothelial activation and are correlated with SM and risk of death ${ }^{22,23}$. Ang2 competes with Ang1 for binding to the receptor tyrosine kinase Tie2, which upon Ang2 binding decreases endothelial barrier integrity. The auto-/paracrine signaling initiated by Ang2 is involved in the transmigration of leukocytes by destabilizing the endothelium ${ }^{24,25}$.

In addition to the presence on leukocytes, ADAM17 is also found along with Ang2 in the WPBs of endothelial cells $^{26}$. Little is known about ADAM17 in malaria, but the cleavage of host receptors specifically utilized by IEs in severe malaria patients and cytokines, such as TNF $\alpha$, which are increased in SM, suggests ADAM17 plays a role in malaria pathogenesis. This study investigated the plasma levels of ADAM17 and Ang2 in Tanzanian children admitted to the hospital with UM, SM, or with other infections, and the potential correlations between ADAM17 or Ang2 and PfEMP1 subtype expression.

\section{Results}

Study population. Characteristics of the study population are presented in Table 1. Children with CM, SMA, RD, hyperparasitaemia were diagnosed as severe malaria (SM). The patients in UM and SM groups were of equal age distributions, the children hospitalized with infections other than malaria were of younger age $(\mathrm{P}<0.0001$, Table 1$)$, while the children with asymptomatic malaria infections were older $(\mathrm{P}=0.02$, Table 1$)$.

Plasma ADAM17 is elevated in hospitalized children. To determine whether ADAM17 plasma levels were associated with clinical malaria, we measured ADAM17 plasma levels by ELISA. Children with UM had a median ADAM17 plasma level of 2,329 pg/mL, and children with SM had a similar median of 2,423 pg/mL (Fig. 1a). Asymptomatic children had lower levels of ADAM17 than children with UM or SM with a median of $490 \mathrm{pg} / \mathrm{ml}(\mathrm{P}=0.04$ and $\mathrm{P}=0.01$, respectively). The median ADAM17 level for the non-malaria patient group was $2,418 \mathrm{pg} / \mathrm{ml}$, similar to UM and SM patients. We observed no difference in the ADAM17 levels between UM and SM patients of Korogwe and Magu. The non-malaria patient group had a higher median level than the asymptomatic children $(\mathrm{P}=0.03)$. Plasma levels of ADAM17 during acute malaria were compared to plasma levels during recovery in samples collected at admission from hospitalized children (Table 1) and from the same children between 2 to 12 weeks after admission (Fig. 1b). In the plasma from convalescent patients, the ADAM17 levels were significantly decreased to a median of $1,180 \mathrm{pg} / \mathrm{ml}(\mathrm{P}<0.0001)$. There was no statistically significant difference between ADAM17 levels in convalescent patients and the asymptomatic malaria children $(\mathrm{P}=0.2)$, and ADAM17 plasma levels samples were negatively correlated with the time progressed since diagnosis (Fig. 1b, $\mathrm{P}=0.0001$ ). Among non-malaria patients, the ADAM17 levels did not differ significantly between the following three groups; septicemia and/or meningitis, pneumonia or submandibular swelling, and those with gastroenteritis or urinary tract infections $(P=0.3$, Fig. S1a). ADAM17 levels were also measured in the plasma from 33 healthy Danish adults (median of $377 \mathrm{pg} / \mathrm{ml}$ ) and were similar to the levels found in the asymptomatic children (Supplementary Fig. S2a).

Ang2 plasma levels are elevated in children with severe malaria. Ang2 plasma levels have previously been shown to correlate with the severity of a malaria infection ${ }^{23}$. We found that Ang2 levels were significantly higher in SM patients compared with UM patients (SM median 2,195 pg/ml vs. UM median 1,461 pg/ml, 


\begin{tabular}{|c|c|c|c|c|c|}
\hline Patient characteristics & $\begin{array}{l}\text { Village infections } \\
(V I)(n=6)\end{array}$ & $\begin{array}{c}\text { Uncomplicated } \\
\text { malaria }(U M)(n=39)\end{array}$ & $\begin{array}{l}\text { Severe malaria }(S M) \\
\quad(n=123)\end{array}$ & $\begin{array}{c}\text { Non-malaria } \\
\text { hospitalized }(n=32)\end{array}$ & $\begin{array}{c}{ }^{*} \text { Acute SM/ } \\
\text { Convalescent }(n=19)\end{array}$ \\
\hline Age (years), mean $[I Q R]$ Min-max & $\begin{array}{l}9.4[5.8-11.5] \\
5.2-16.9\end{array}$ & $3.0[2.0-4.0] 0.2-7.0$ & $2.8[1.6-4.0] 0.1-9.2$ & $1.5[0.6-2.2] 0.2-4.9$ & $2.5[1.3-3.5] 0.3-4.2$ \\
\hline $\operatorname{Sex}, n(M / F)$ & $3 / 3$ & $16 / 23$ & $60 / 63$ & $21 / 11$ & $10 / 9$ \\
\hline Location, $n$ (Korogwe/Magu) & NA & $32 / 7$ & $75 / 48$ & $32 / 0$ & $19 / 0$ \\
\hline Parasitaemia, median $(p / \mu L)[I Q R]$ & $989[363-1,650]$ & $\begin{array}{c}12,500 \\
{[5,080-45,000]}\end{array}$ & $\begin{array}{c}272,000 \\
{[35,000-512,200]}\end{array}$ & NA & $\begin{array}{c}17,000 \\
{[9,900-427,000]}\end{array}$ \\
\hline HRP2, median $(\mu g / m L)[I Q R]$ & $435[158-3371]$ & $1662[92-3215]$ & $3989[868-8013]$ & $0[0-0]$ & $1715[1020-2380]$ \\
\hline Temperature, median $\left({ }^{\circ} \mathrm{C}\right)[I Q R]$ & $37.1[36.8-38.2]$ & $38.0[37.6-39.7]$ & $38.4[37.7-39.4]$ & $39.3[38.9-39.8]$ & $38.8[38.1-39.1]$ \\
\hline Haemoglobin, mean $\pm s d(g / d L)$ Min-max & $11.3 \pm 2.66 .7-14.7$ & $9.9 \pm 2.08 .1-20.2$ & $6.16 \pm 2.32 .4-11.3$ & $9.1 \pm 1.65 .6-12.2$ & $5.9 \pm 2.96 .7-14.7$ \\
\hline Blantyre coma score, mean $[I Q R]$ Min-max & NA & $5[5-5] 5-5$ & $3.6[2-5] 0-5$ & $4.8[5-5] 1-5$ & $4.5[5-5] 2-5$ \\
\hline Lactate, mean $\pm s d(m M)$ Min-max & NA & $3.4 \pm 1.81 .4-9.0$ & $5 \pm 3.40 .9-17.2$ & $3.1 \pm 1.21 .5-6.1$ & $5.5 \pm 3.11 .1-13.1$ \\
\hline Mortality, $n(\%)$ & 0 & 0 & $9(7)$ & $1(3)$ & 0 \\
\hline
\end{tabular}

Table 1. Clinical characteristics of patients. ${ }^{*}$ For 19 SM cases a convalescent plasma sample was taken. $\mathrm{IQR}=$ Inter Quartile Range, sd = Standard deviation.

$\mathrm{P}<0.0001$, Fig. 2). The median Ang2 plasma levels in individuals with asymptomatic malaria (median $926 \mathrm{pg} / \mathrm{ml}$ ) or hospitalized with non-malaria infections (median $884 \mathrm{pg} / \mathrm{ml}$ ) were both significantly lower than in the SM group $(\mathrm{P}<0.0001)$. There was no statistically significant difference between the Ang2 levels of those with UM and asymptomatic malaria. Within the non-malaria groups, the Ang2 levels did not differ significantly between the different non-malaria cases $(\mathrm{P}=0.4$, Supplementary Fig. S1b). In plasma from healthy Danish adults we found a median Ang2 level of $576 \mathrm{pg} / \mathrm{ml}$ (Supplementary Fig. S2b). When looking at Magu and Korogwe individually, both had an increased Ang2 in SM compared to UM ( $\mathrm{P}=0.0002$ and $\mathrm{P}=0.0004$, respectively). The levels of Ang2 in SM patients were higher in Magu compared with Korogwe $(\mathrm{P}<0.0001)$, while the levels of Ang2 in UM patients did not differ significantly $(\mathrm{P}=0.1)$

Ang2 but not ADAM17 plasma levels are associated with malaria severity in hospitalized children. As we found no difference in ADAM17 levels between the UM and SM groups, these were grouped for the further analysis of factors or variables correlated with the ADAM17 levels in patients hospitalized with malaria. We found no correlation with any of the symptoms or signs previously associated with increased risk of fatal outcome of a malaria infection and plasma ADAM17 levels, such as low Hb, high lactate, or Blantyre coma score $<3$. ADAM17 levels were correlated with Ang2 $(P=0.002$, Table 2), weakly correlated with the marker for parasite biomass HRP2 (although not statistically significant when correcting for multiple testing, Rho $=0.17$, $\mathrm{P}=0.03)$, and ADAM17 levels were inversely correlated with age $(\mathrm{P}=0.001)$. By contrast, Ang2 plasma levels were inversely correlated with Blantyre coma score $(\mathrm{P}=0.0002)$ and Hb-levels $(\mathrm{P}<0.0001)$ and positively correlated with lactate $(\mathrm{P}<0.0001)$, but had no significant correlation with age or parasitaemia (both when measured as circulating erythrocytes or by analyzing plasma levels of HRP2) (Table 2).

Ang2 but not ADAM17 plasma levels are associated with PfEMP1 type expression. IE sequestration via the PfEMP1-EPCR interaction is thought to affect pathology through occlusion of blood flow and promotion of coagulation and inflammation ${ }^{27}$.

We utilized var transcript profiles of the UM and SM individuals (Mkumbaye et al., submitted), to explore Ang2 and ADAM17 plasma level correlations with expression of specific var subsets, encoding PfEMP1 predicted to bind or not to bind EPCR. Group A PfEMP1 expression levels were estimated by the "DBLalall" primer set. The DBLalall reported expression levels that correlated with Ang2 plasma levels but not with ADAM17 plasma levels (Table 2). Group A transcripts targeted by the DBLa2/1.1/2/4/7 primers typically encode EPCR-binding CIDR $\alpha 1$ domains ${ }^{7}$. Abundance of such transcripts also weakly correlated with Ang2 (Rho $\left.=0.25, \mathrm{P}=0.002\right)$, whereas group A transcripts encoding PfEMP1 not binding EPCR (reported by the DBLa1.5/6/8 primers) were not correlated with Ang2 plasma levels $(\mathrm{P}=0.27)$ (Table 2). When investigating transcript levels reported by primers specific for genes encoding different EPCR-binding CIDR $\alpha 1$ subtypes, we found a weak correlation between plasma levels of Ang2 and the abundance of transcripts encoding CIDR $\alpha 1.1$ (DC8) (Rho $=0.25$, $\mathrm{P}=0.001$, Table 2) and CIDR $\alpha 1.4$ (DC13) domains, although CIDR $\alpha 1.4$ was not statistically significant when using Bonferroni's correction for multiple testing ( $\mathrm{Rho}=0.18, \mathrm{P}=0.02$; Table 2). ADAM17 plasma levels were weakly correlated with the abundance of CIDR $\alpha 1.1$ encoding transcripts, however, this correlation was not statistically significant with Bonferroni's correction $(\mathrm{Rho}=0.18, \mathrm{P}=0.02$; Table 2$)$. We observed no statistically significant correlation between expression of PfEMP1 containing CIDR $\alpha 3.1$ and CIDR $\alpha 3.2$ predicted to bind CD36 (primer CIDRa3.1/2) and Ang2 plasma levels or ADAM17 plasma levels (Table 2).

Next, we evaluated the Ang2 plasma level in relation to the correlated components. As the Bonferroni's correction applied in the univariate analysis is quite conservative, we chose to include all variables correlated with a $\mathrm{P}$ value below 0.05 to include testing of possible false negatives, $\mathrm{Hb}$, lactate, parasitaemia, Blantyre coma score, CIDR $\alpha 1.4$, CIDR $\alpha 1.1$, and ADAM17 in a general multiple linear regression analysis with backwards elimination of covariates (controlled for age and location). We found that $\mathrm{Hb}$, lactate, CIDR $\alpha 1.4$, and ADAM17 were 
a

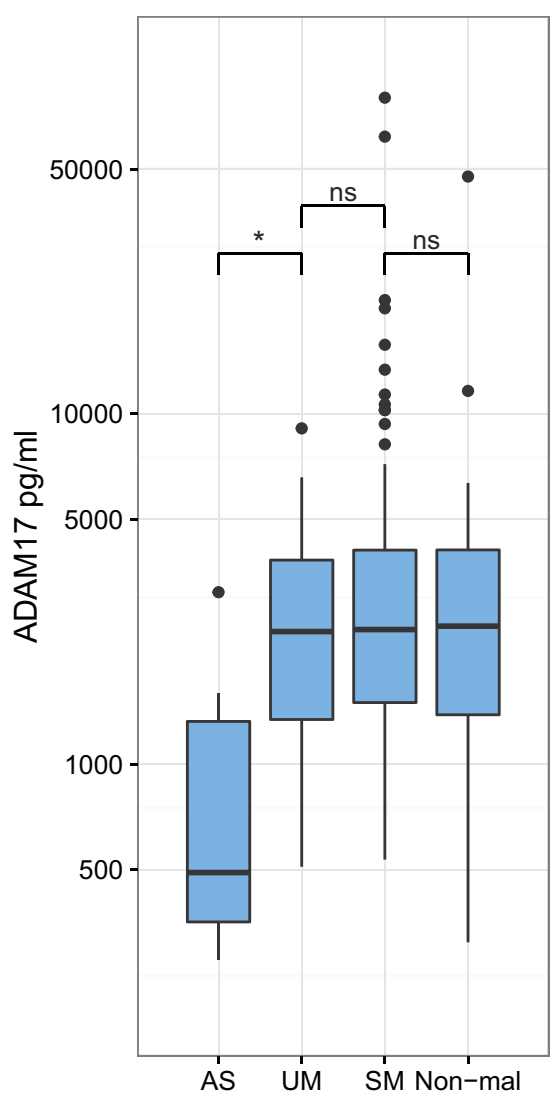

b

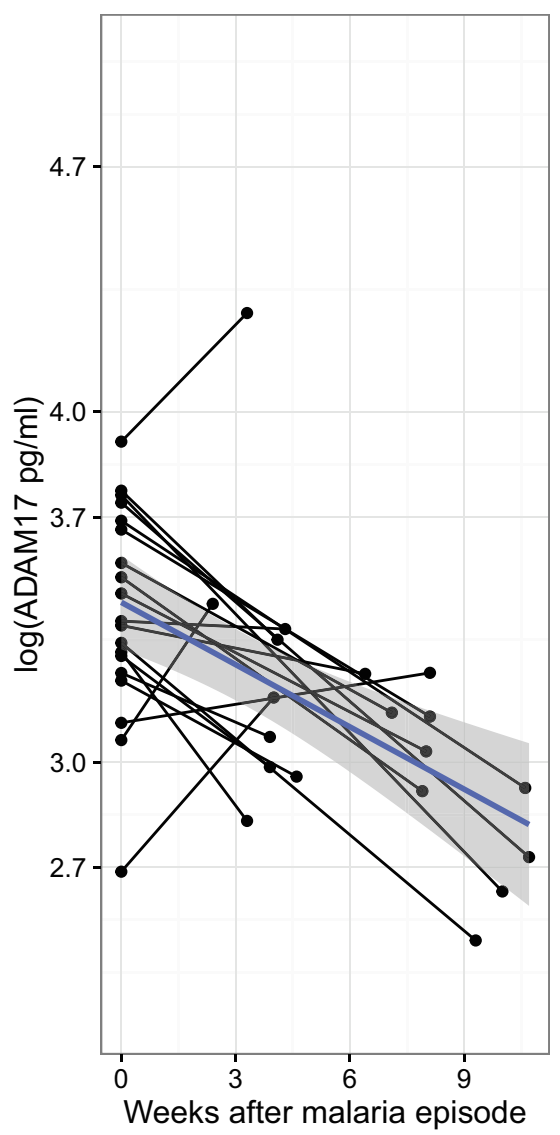

Figure 1. ADAM17 plasma levels. (a) Boxplot of ADAM17 plasma levels for children with either asymptomatic malaria (AS, $n=6)$, uncomplicated malaria $(U M, n=39)$, severe malaria $(S M, n=123)$, or children admitted to the hospital without malaria (Non-mal, $n=32$ ). The boxplots display median, $1^{\text {st }}$ quartile (Q1), and $3^{\text {rd }}$ quartile (Q3). The lower whisker indicates the lowest data point above Q1 minus 1.5 times IQR, the upper whisker indicates the highest data point below 1.5 IQR plus Q3. Values below Q1 minus 1.5 IQR and above Q3 plus 1.5 IQR are indicated as points. Statistical significance was calculated using Kruskal-Wallis test and pairwise comparisons using Dunn's Test with Bonferroni correction. ${ }^{\star} \mathrm{P}<0.05$. (b) Acute/convalescent plasma levels of ADAM17 samples from 19 children hospitalized with malaria. Acute samples taken at $\mathrm{t}=0$, convalescent follow-up samples were taken between 2 and 12 weeks after the hospitalization. ADAM17 data is log-transformed. Linear model in blue with $95 \%$ confidence interval in grey (P-value $=0.0001)$.

statistically significant explanatory variables for Ang2 plasma levels (Table 3). We evaluated the correlations found between ADAM17 plasma levels and age, HRP2, Ang2 plasma levels, CIDR $\alpha 3.1 / 2$ expression, and expression of CIDR $\alpha 1.1$ in a general multiple linear regression analysis with backwards elimination of covariates. We found that CIDR $\alpha 1.1$ expression was not a statistically significant explanatory variable for ADAM17 levels, while age and Ang2 levels were statistically significant explanatory variables (Table 4).

\section{Discussion}

The pathology of malaria is the result of a complex interplay between inflammatory response, endothelial activation, parasite sequestration, and loss of erythrocytes. An increased amount of cytokines released by ADAM17 and endothelial activation with associated increase in plasma levels of Ang2 have been reported in malaria patients ${ }^{23}$. Parasites expressing EPCR-binding PfEMP1 are associated with $\mathrm{SM}^{6,7,28}$, and expression of group A PfEMP1s (EPCR and non-EPCR-binding PfEMP1) has been correlated with increased Ang2 plasma levels ${ }^{29}$.

In this study, we investigated the plasma levels of ADAM17 and Ang2 in children in north-eastern and north-western Tanzania hospitalized with malaria infections in relation to PfEMP1 expression, and the clinical parameters; Hb, lactate, Blantyre coma score, and parasitaemia. We found ADAM17 plasma levels elevated in hospitalized children with malaria compared with children with asymptomatic malaria infections. However, the ADAM17 plasma levels were similar between children with UM, SM, and other infectious diseases. During malaria convalescence, ADAM17 plasma levels markedly declined to levels similar to those of asymptomatic malaria-infected children. The increased ADAM17 concentrations in the plasma may be part of a general inflammatory response to infection and seemed to be unspecific. 




Figure 2. Angiopoietin 2 (Ang2) plasma levels. Boxplot of Ang2 plasma levels for children with either asymptomatic malaria (AS, $n=6)$, uncomplicated malaria $(U M, n=39)$, severe malaria $(S M, n=123)$, or children admitted to the hospital without malaria (Non-mal, $\mathrm{n}=32$ The boxplots display median, $1^{\text {st }}$ quartile $(\mathrm{Q} 1)$, and $3^{\text {rd }}$ quartile (Q3). The lower whisker indicates the lowest data point above Q1 minus 1.5 times IQR, the upper whisker indicates the highest datapoint below 1.5 IQR plus Q3. Values below Q1 minus 1.5 IQR and above Q3 plus 1.5 IQR are indicated as points. Statistical significance was calculated using Kruskal-Wallis test and pairwise comparisons using Dunn’s Test with Bonferroni correction. ${ }^{* *} \mathrm{P}<0.0001$.

In line with previous studies, Ang2 plasma levels were increased in SM patients compared with UM patients and non-malarial patients ${ }^{23,29}$. Ang2 levels in SM cases appeared higher in Magu than in Korogwe, while the UM cases had similar Ang2 levels. Ang2 levels, unlike ADAM17, correlated with markers of disease severity, such as Blantyre coma score, $\mathrm{Hb}$ - and lactate levels in children hospitalized with malaria. We also observed a weak correlation between Ang2 and ADAM17. Ang2 is released to the plasma from WPBs of activated endothelial cells $^{21}$, and ADAM17 is co-localized with Ang2 in the $\mathrm{WBPs}^{26}$, which could explain this correlation. However, as ADAM17 plasma levels seem to be elevated as a response to disease in general, whereas Ang2 plasma levels are particularly increased in SM, it seems unlikely that the increase of ADAM17 plasma levels was primarily due to activation of endothelial cells and subsequent release of WPB. ADAM17 sheds EPCR, and loss of EPCR from the endothelial cell surface or of EPCR function may lead to a rise of plasma Ang2 ${ }^{30}$. However, the origin of ADAM17 in plasma is unknown. Secretion of enzymatically active ADAM17 on extracellular microparticles has been found in the plasma and associated with disease activity of anti-neutrophil cytoplasmic antibody (ANCA)-associated vasculitis $^{26}$. ANCA-associated vasculitis, as malaria, features an excessive inflammatory response, which leads to the shedding of adhesion molecules and cytokines, such as $\mathrm{TNF}^{31,32}$.

ADAM17 sheds EPCR, and thus the increased plasma ADAM17 during acute malaria infections may have consequences for the survival of EPCR-binding parasites and for regulation of inflammation, including increased levels of plasma Ang2. However, ADAM17 plasma levels were not associated with reduced expression 


\begin{tabular}{|l|r|l|c|c|}
\hline \multirow{2}{*}{ Age } & \multicolumn{2}{|c|}{ ADAM17 } & \multicolumn{2}{c|}{ Ang2 } \\
\cline { 2 - 5 } & \multicolumn{1}{|c|}{ Rho } & P value & Rho & P value \\
\hline Hb & -0.24 & $0.0013^{*}$ & -0.014 & 0.86 \\
\hline Lactate & -0.10 & 0.15 & -0.39 & $0.0000006^{*}$ \\
\hline Parasitaemia & 0.08 & 0.34 & 0.35 & $0.0001^{*}$ \\
\hline HRP2 & -0.05 & 0.50 & 0.15 & 0.06 \\
\hline Blantyre score & 0.17 & 0.03 & 0.07 & 0.42 \\
\hline Ang2 & -0.02 & 0.84 & -0.29 & $0.0002^{*}$ \\
\hline DBLa1all & 0.18 & 0.02 & & \\
\hline DBLa2/1.1/2/4/7 & 0.1 & 0.21 & 0.24 & $0.002^{*}$ \\
\hline DBLa1.5/6/8 & 0.10 & 0.19 & 0.25 & $0.002^{*}$ \\
\hline CIDRa1.all & 0.09 & 0.23 & 0.09 & 0.27 \\
\hline CIDRa1.A & 0.08 & 0.34 & 0.17 & 0.04 \\
\hline CIDRa1.4 & -0.03 & 0.75 & 0.18 & 0.02 \\
\hline CIDRa1.5 & 0.02 & 0.73 & 0.18 & 0.02 \\
\hline CIDRa1.6 & -0.08 & 0.31 & 0.05 & 0.50 \\
\hline CIDRa1.7 & -0.03 & 0.71 & 0.07 & 0.37 \\
\hline CIDRa1.DC8 & -0.09 & 0.23 & 0.08 & 0.28 \\
\hline CIDRa1.1 & 0.08 & 0.28 & 0.15 & 0.06 \\
\hline CIDRa1.8 & 0.18 & 0.02 & 0.25 & $0.001^{*}$ \\
\hline CIDRa3.1/2 & -0.04 & 0.56 & 0.07 & 0.40 \\
\hline UPSB & 0.15 & 0.05 & 0.10 & 0.23 \\
\hline VAR2CSA & 0.03 & 0.69 & 0.03 & 0.72 \\
\hline Var3 & -0.11 & 0.16 & -0.02 & 0.78 \\
\hline CIDRd & 0.13 & 0.12 & 0.02 & 0.83 \\
\hline CIDRg & 0.09 & 0.21 & -0.02 & 0.81 \\
\hline DBLz & 0.04 & 0.59 & -0.04 & 0.59 \\
\hline DBLe & 0.03 & 0.71 & 0.10 & 0.19 \\
\hline DC5 & 0.03 & 0.66 & -0.03 & 0.75 \\
\hline & 0.03 & 0.74 & -0.05 & 0.51 \\
\hline & & & & \\
\hline DRA & & & \\
\hline
\end{tabular}

Table 2. Correlations of ADAM17 and Ang2 Plasma levels. *Statistically significant correlations when applying Bonferroni's correction for multiple testing with 24 independent tests for ADAM17 and Ang2 each $(\mathrm{P} \leq 0.002$ considered statistically significant, DBL $\alpha 1$ all, CIDR $\alpha 1$.all, CIDR $\alpha 1$.A, and CIDR $\alpha 1$.DC8 were not counted as they represent summations). CIDR $\alpha 1$.all = Sum of all primers targeting transcripts encoding CIDR $\alpha$ 1 s predicted to bind EPCR. CIDRa1.A EPCR $=$ Sum of all transcript abundances reported by primers targeting genes encoding Group A CIDR $\alpha 1$ s predicted to bind EPCR. CIDR $\alpha 1 . D C 8=$ Sum of all reported transcripts encoding Group B/A CIDR $\alpha 1$ s predicted to bind EPCR. Spearman's rank correlation coefficients (Rho) calculated for uncomplicated and severe acute malaria patients.

\begin{tabular}{|l|c|c|}
\hline Model & Estimate & $\boldsymbol{P}$ \\
\hline $\mathrm{Hb}(\mathrm{g} / \mathrm{dL})$ & -0.03 & 0.001 \\
\hline Lactate & 0.02 & 0.004 \\
\hline CIDRo1.4 & 0.003 & 0.004 \\
\hline Log10 $(\mathrm{ADAM} 17)(\mathrm{pg} / \mathrm{ml})$ & 0.15 & 0.02 \\
\hline Location 2 & 0.2 & 0.0001 \\
\hline
\end{tabular}

Table 3. Statistically significant explanatory variables in a multiple linear regression analysis of transformed Ang2 levels in hospitalized malaria patients.

\begin{tabular}{|l|c|c|}
\hline Model & Estimate & $\boldsymbol{P}$ \\
\hline Age $($ years $)$ & -0.05 & 0.002 \\
\hline Log10 $($ Ang2 pg/ml $)$ & 0.25 & 0.005 \\
\hline
\end{tabular}

Table 4. Statistically significant explanatory variables in a multiple linear regression analysis of transformed ADAM17 levels in hospitalized malaria patients. 
of EPCR-binding PfEMP1. Rather, expression of PfEMP1 with CIDR $\alpha 1.1$ domains was weakly correlated with ADAM17 plasma levels, although this correlation was not statistically significant when correcting for multiple testing, and lost after adjusting for age by general multiple linear regression analysis. This suggests that PfEMP1 expression and ADAM17 levels in the plasma are not directly affecting each other during malaria.

Previously, Abdi et al., showed that Ang2 plasma levels were associated with group A-like PfEMP1 expression and rosetting ${ }^{29}$, and that Ang2 levels were associated with development of the specific SM clinical manifestations $\mathrm{RD}$ and CM. A-type PfEMP1 expression was only associated with CM and not RD, and Abdi et al. concluded that Ang2 plasma levels and group A-like PfEMP1 expression were independently associated with impaired consciousness and $\mathrm{CM}^{29}$. Using a comprehensive set of var type specific primers we found that Ang2 levels are specifically correlated with expression of var genes encoding PfEMP1s predicted to bind EPCR. DBL 1 domain expression measured with primers targeting group A PfEMP1s (DBL 1 1all) correlated with Ang2, similarly to what Abdi et al. reported, while further division of the group A showed that only the subtype of group A (targeted by primer DBL $\alpha 2 / 1.1 / 4 / 7$ ) genes predicted to bind EPCR correlated with Ang2, and expression of group A PfEMP1 predicted not to bind EPCR did not correlate with Ang2. Furthermore, measurements of additional var genes encoding PfEMP1s not predicted to bind EPCR, including group B PfEMP1 (UPSB), PfEMP1 containing CIDR $\alpha 3.1 / 2$ predicted to bind CD36 or chondroitin sulfate A (VAR2CSA), did not correlate with plasma levels of Ang2.

We did not find a correlation between the transcript levels of all analysed CIDR $\alpha 1$ domains predicted to bind EPCR, so caution is warranted in interpreting the found correlations, but it could be due to less prevalence of CIDR $\alpha 1$ expression of these subtypes in the infecting parasites. Our measurements of PfEMP1 expression are not able to fully account for the increase in Ang2 observed in SM, and cannot alone explain the increase in Ang2 observed in SM, as underlined by our multiple linear regression analysis of Ang2 levels. So while Abdi et al. reports that endothelial activation and group A expression are independently associated with CM, we find that part of the Ang2 plasma level which is increased in SM, is associated with EPCR-binding PfEMP1 expression, even when including other factors associated with increased Ang2 in the analysis, such as lactate and $\mathrm{Hb}$.

Recently, an analysis of full-length var transcripts in UM and SM groups revealed that they mainly differ in relation to an increased expression of EPCR-binding PfEMP1 in SM, but with no major differences in var transcripts between SMA and CM groups ${ }^{33}$.

In summary, these data suggest that ADAM17 plasma levels increase in response to malaria infection similarly to that of other infectious diseases and that the association between Ang2 levels and var gene transcript levels, although weak, is specific for EPCR-binding PfEMP1s. Furthermore, PfEMP1s predicted to bind EPCR may be associated with the endothelial activation accompanying SM, reinforcing the notion that EPCR-binding PfEMP1s are linked to SM pathogenesis (Supplementary Fig. S3).

\section{Methods}

Sample collection. A total of 213 children admitted to Korogwe and Magu District Hospitals, in north-eastern and north-western Tanzania, respectively, were enrolled as part of an ongoing monitoring of malaria at the hospitals. 181 children were blood smear positive for P. falciparum. For 19 children admitted to the Korogwe District Hospital with malaria, blood samples were drawn at admission and at follow-up between 2 and 12 weeks after admission. None were malaria positive at follow-up by rapid diagnostic tests. 32 of the children admitted to Korogwe District Hospital with infectious diseases other than malaria were included as non-malaria hospitalized children and also a part of a previous study ${ }^{34}$ (Supplementary Table S1). The patient samples included in this study were selected from a database to reflect the main clinical manifestations associated with high mortality; (I) CM was diagnosed if the Blantyre coma score was $<3^{35}$ ), (II) SMA if the haemoglobin ( $\mathrm{Hb}$ ) level was $<5 \mathrm{~g} / \mathrm{dl}$, (III) if there were clinical signs of RD (Kussmaul deep breathing and/or chest indrawing), or (IV) if hyperparasitaemia was observed $(>200,000$ parasites/ $\mu \mathrm{l})$. Children fulfilling any of the above criteria were categorized as SM. Children not fulfilling any of these criteria were categorized as UM. Plasma samples from six non-hospitalized children as part of a malariometric survey in the Korogwe district with asymptomatic malaria were included in the study.

Ethics statement. Written informed consent was obtained from the parents or guardian of all the study participants. All patients received treatment according to the national guidelines. The studies and experiments were reviewed and approved by the National Institute for Medical Research, Tanzania (reference no. NIMR/HQ/R.8c/ Vol.II/436), and the methods in this study were perfomed in accordance with the approved guidelines.

Detection of plasma proteins. The plasma samples were diluted in phosphate buffered saline (PBS) + 1\% bovine serum albumin (BSA), between 1:2 and 1:6 for ADAM17 and 1:5 for Ang2, and ADAM17 and Ang2 plasma levels were detected using DuoSet ELISA Development kit (R\&D Systems) according to manufacturer's instructions. A four parameter logistic curve was fitted to a serial dilution of a recombinant protein standard of known concentration for each ELISA plate, in order to convert optical densities to protein concentration.

The PfHRP2 plasma levels were determined using an ELISA assay as previously described ${ }^{36}$, with the following modifications: Maxisorp plates (NUNC, Roskilde, Denmark) were coated with $1.0 \mu \mathrm{g} / \mathrm{mL}$ immunoglobulin M monoclonal anti-HRP2 antibody (MPFM-55a, Immunology Consultants Laboratories, Portland, USA) diluted in PBS and incubated overnight at $4{ }^{\circ} \mathrm{C}$. Plates were blocked with $3 \%$ skimmed milk in PBS for 2 hours. Between each step the plates were washed in $0.05 \%$ Tween-20 and PBS. Plasma samples were diluted 1:64 in 1\% skimmed milk and PBS, added to the plates and incubated for 2 hours. Secondary antibody conjugated with horseradish peroxidase (MPFG-55P, Immunology Consultants Laboratories; $0.2 \mu \mathrm{g} / \mathrm{mL}$ diluted in $1 \%$ skimmed milk and PBS) was added to each well and incubated for 1 hour at room temperature. Substrate, TMB ease (Kem-En-Tec Diagnostics), was added and the reaction was stopped with $0.2 \mathrm{M} \mathrm{H}_{2} \mathrm{SO}_{4}$. The plates were read using a plate reader 
at an OD of 490. A serial dilution of a recombinant HRP2 protein (Cat no MBS5303860, MyBioSource, San Diego, USA) was made for each plate in order to convert optical densities to protein concentration as for ADAM17 and Ang2.

Var transcript abundances. Data of transcript abundances of var gene subtypes were generated using quantitative PCR by Mkumbaye et al., (submitted) as previously described ${ }^{7}$, although using redesigned primers targeting loci specific for different PfEMP1 domains. The qPCR primer sets were optimized for optimal coverage of var genes encoding specific subclasses of DBL and CIDR domains. Supplementary Table S2 provides an overview of the estimated var type coverage of the primers used in this study. qPCR was performed in $20-\mu \mathrm{L}$ reactions using QuantiTect SYBR Green PCR master mix (Qiagen) with the Rotorgene thermal cycler system (Corbett Research), and the transcript abundance was determined relative to the averaged transcript abundance of seryl-tRNA synthetase and fructose-bisphosphate aldolase, as previously described ${ }^{7}$. Summation of var transcript levels reported by primers targeting var transcripts encoding PfEMP1 with similar receptor binding specificity was done for: PfEMP1 with CIDR $\alpha 1$ domains predicted to bind EPCR (CIDRa1.all): primer sets CIDRa1.1, CIDRa1.4, CIDRa1.5, CIDRa1.6, CIDRa1.7, and CIDRa1.8; Group A CIDR $\alpha 1$ domains predicted to bind EPCR (CIDRa1.A): primer sets CIDRa1.4, CIDRa1.5, CIDRa1.6, and CIDRa1.7; and DC8 (CIDR $\alpha 1 . D C 8)$ : primer sets CIDRa1.1 and CIDRa1.8.

Statistical analysis. Normally distributed variables, such as age, were compared by Welch's Two Sample t-test. For comparing acute and convalescent ADAM17 plasma levels, Wilcoxon's paired signed rank test was used to calculate statistical significance. For comparisons of multiple groups, the non-parametric Kruskal-Wallis test was used, while differences between groups were calculated by pairwise comparisons using Dunn's test with Bonferroni's correction. For univariate correlations, Spearman's rank correlation was used to determine the correlation coefficient Rho. Bonferroni's correction was applied to the spearman's rank correlations; ADAM17 and Ang2 were considered independent tests, so 24 components were included in the correction for each (DBL $\alpha$ 1all, CIDR $\alpha 1$.all, CIDR $\alpha 1$.A, and CIDR $\alpha 1$.DC8 were not counted as they represent summations of primers that are included by themselves) and thus correlations were considered statistically significant when the P-value was below 0.002. General linear regression analysis was performed, in which we included the univariate correlations with a $P$ value below 0.05 . Backwards elimination was performed with multiple iterations, each time removing the variable with the highest P-value above the significance level, until only statistically significant explanatory variables remained. Statistical analyses were performed using the statistical software R. A P-value below 0.05 was considered statistically significant.

\section{References}

1. World Health Organization. World Malaria Report 2015. World Health, doi: ISBN 9789241564403 (2015).

2. Miller, L. H., Baruch, D. I., Marsh, K. \& Doumbo, O. K. The pathogenic basis of malaria. Nature 415, 673-679 (2002).

3. Turner, L. et al. Severe malaria is associated with parasite binding to endothelial protein C receptor. Nature 498, 502-505 (2013).

4. Lavstsen, T., Salanti, A., Jensen, A. T. R., Arnot, D. E. \& Theander, T. G. Sub-grouping of Plasmodium falciparum 3D7 var genes based on sequence analysis of coding and non-coding regions. Malar. J. 2, 27 (2003).

5. Rask, T. S., Hansen, D. A., Theander, T. G., Gorm Pedersen, A. \& Lavstsen, T. Plasmodium falciparum erythrocyte membrane protein 1 diversity in seven genomes-divide and conquer. PLoS Comput. Biol. 6, e1000933 (2010).

6. Lau, C. K. Y. et al. Structural conservation despite huge sequence diversity allows EPCR binding by the pfemp 1 family implicated in severe childhood malaria. Cell Host Microbe 17, 118-129 (2015).

7. Lavstsen, T. et al. Plasmodium falciparum erythrocyte membrane protein 1 domain cassettes 8 and 13 are associated with severe malaria in children. Proc. Natl. Acad. Sci. 109, E1791-E1800 (2012).

8. Avril, M. et al. A restricted subset of var genes mediates adherence of Plasmodium falciparum-infected erythrocytes to brain endothelial cells. Proc. Natl. Acad. Sci. 109, E1782-E1790 (2012).

9. Claessens, A. \& Rowe, J. A. Selection of Plasmodium falciparum parasites for cytoadhesion to human brain endothelial cells. J. Vis. Exp. e3122, doi: 10.3791/3122 (2012).

10. Wajant, H., Pfizenmaier, K. \& Scheurich, P. Tumor necrosis factor signaling. Cell Death Differ. 10, 45-65 (2003).

11. Sundgren-Andersson, A. K., Ostlund, P. \& Bartfai, T. IL-6 is essential in TNF-alpha-induced fever. Am. J. Physiol. 275, R2028-R2034 (1998).

12. Kwiatkowski, D. et al. TNF concentration in fatal cerebral, non-fatal cerebral, and uncomplicated Plasmodium falciparum malaria. Lancet 336, 1201-1204 (1990).

13. Moss, M. L. et al. Structural features and biochemical properties of TNF-alpha converting enzyme (TACE). J. Neuroimmunol. 72, 127-129 (1997)

14. Scheller, J., Chalaris, A., Garbers, C. \& Rose-John, S. ADAM17: A molecular switch to control inflammation and tissue regeneration. Trends in Immunology 32, 380-387 (2011)

15. Tsakadze, N. L. et al. Tumor necrosis factor-alpha-converting enzyme (TACE/ADAM-17) mediates the ectodomain cleavage of intercellular adhesion molecule-1 (ICAM-1). J. Biol. Chem. 281, 3157-3164 (2006).

16. Qu, D., Wang, Y., Esmon, N. L. \& Esmon, C. T. Regulated endothelial protein C receptor shedding is mediated by tumor necrosis factor-? converting enzyme/ADAM17. J. Thromb. Haemost. 5, 395-402 (2007).

17. Moxon, C. A. et al. Loss of endothelial protein C receptors links coagulation and inflammation to parasite sequestration in cerebral malaria in African children. Blood 122, 842-851 (2013).

18. Sampath, S. et al. Plasmodium falciparum adhesion domains linked to severe malaria differ in blockade of endothelial protein C receptor. Cell. Microbiol. 17, 1868-1882 (2015).

19. Petersen, J. E. V. et al. Protein C system defects inflicted by the malaria parasite protein PfEMP1 can be overcome by a soluble EPCR variant. Thromb. Haemost. 114, 1038-1048 (2015).

20. Gillrie, M. R. et al. Diverse functional outcomes of Plasmodium falciparum ligation of EPCR: Potential implications for malarial pathogenesis. Cell. Microbiol. 17, 1883-1899 (2015).

21. Fiedler, U. et al. The Tie-2 ligand Angiopoietin-2 is stored in and rapidly released upon stimulation from endothelial cell WeibelPalade bodies. Blood 103, 4150-4156 (2004)

22. Lovegrove, F. E. et al. Serum angiopoietin-1 and -2 levels discriminate cerebral malaria from uncomplicated malaria and predict clinical outcome in African children. PLoS One 4 (2009). 
23. Yeo, T. W. et al. Angiopoietin-2 is associated with decreased endothelial nitric oxide and poor clinical outcome in severe falciparum malaria. Proc. Natl. Acad. Sci. USA 105, 17097-17102 (2008).

24. Hakanpaa, L. et al. Endothelial destabilization by angiopoietin-2 via integrin $\beta 1$ activation. Nat. Commun. 6, 5962 (2015).

25. Dreymueller, D. et al. Lung endothelial ADAM17 regulates the acute inflammatory response to lipopolysaccharide. EMBO Mol. Med. 4, 412-423 (2012).

26. Bertram, A. et al. Circulating ADAM17 Level Reflects Disease Activity in Proteinase-3 ANCA-Associated Vasculitis. J. Am. Soc. Nephrol, doi: 10.1681/ASN.2014050477 (2015).

27. Miller, L. H., Ackerman, H. C., Su, X. \& Wellems, T. E. Malaria biology and disease pathogenesis: insights for new treatments. Nat. Med. 19, 156-167 (2013).

28. Turner, L. et al. Severe malaria is associated with parasite binding to endothelial protein C receptor. Nature 498, 502-505 (2013).

29. Abdi, A. I. et al. Plasmodium falciparum antigenic variation: relationships between widespread endothelial activation, parasite PfEMP1 expression and severe malaria. BMC Infect. Dis. 14, 170 (2014).

30. Bae, J. S. \& Rezaie, A. R. Thrombin upregulates the angiopoietin-Tie2 Axis: Endothelial protein C receptor occupancy prevents the thrombin mobilization of angiopoietin 2 and P-selectin from Weibel-Palade bodies. J. Thromb. Haemost. 8, 1107-1115 (2010).

31. Ohlsson, S., Wieslander, J. \& Segelmark, M. Circulating cytokine profile in anti-neutrophilic cytoplasmatic autoantibody-associated vasculitis: prediction of outcome? Mediators Inflamm. 13, 275-283 (2004).

32. Noronha, I. L., Krüger, C., Andrassy, K., Ritz, E. \& Waldherr, R. In situ production of TNF-alpha, IL-1 beta and IL-2R in ANCApositive glomerulonephritis. Kidney Int. 43, 682-692 (1993).

33. Jespersen, J. S. et al. Plasmodium falciparum var genes expressed in children with severe malaria encode CIDR $\alpha 1$ domains. EMBO Mol. Med. 8, 839-850 (2016).

34. Hansson, H. H. et al. Haplotypes of the endothelial protein C receptor (EPCR) gene are not associated with severe malaria in Tanzania. Malar. J. 14, 474 (2015).

35. Molyneux, M. E., Taylor, T. E., Wirima, J. J. \& Borgstein, A. Clinical features and prognostic indicators in paediatric cerebral malaria: a study of 131 comatose Malawian children. Q. J. Med. 71, 441-459 (1989).

36. Kariuki, S. M. et al. Value of plasmodium falciparum histidine-rich protein 2 level and malaria retinopathy in distinguishing cerebral malaria from other acute encephalopathies in Kenyan children. J. Infect. Dis. 209, 600-609 (2014).

\section{Acknowledgements}

We thank the Tanzanian children and parents as well as the Danish adults for participating in the study. The clinical and technical staff of the Joint Malaria Programme at Korogwe District Hospital and at Magu District Hospitals in Tanzania are also thanked. The study received financial support from the Danish International Development Agency, The Danish Council for Independent Research, Medical Sciences grant ID 1333-00220 (CW), Faculty of Health and Medical Sciences, University of Copenhagen (JP), Axel Muusfeldts Foundation, Augustinus Foundation, and Grosserer L.F. Foght Foundation.

\section{Author Contributions}

J.E.V.P., S.I.M. and A.V.V. conducted the experiments. C.W.W., SI.M., J.P.L. and T.L. designed and planned the study. S.I.M., A.M., E.L., R.A.K., S.B.M., J.M., D.T.R.M. and J.P.L. were responsible for collecting the patient samples. J.P.L., S.I.M., C.W.W., T.L. and T.G.T. analysed the data and wrote the manuscript. All authors reviewed the manuscript.

\section{Additional Information}

Supplementary information accompanies this paper at http://www.nature.com/srep

Competing financial interests: The authors declare no competing financial interests.

How to cite this article: Petersen, J. E. V. et al. Plasma Ang2 and ADAM17 levels are elevated during clinical malaria; Ang2 level correlates with severity and expression of EPCR-binding PfEMP1. Sci. Rep. 6, 35950; doi: 10.1038/srep35950 (2016).

Publisher's note: Springer Nature remains neutral with regard to jurisdictional claims in published maps and institutional affiliations.

(c) (i) This work is licensed under a Creative Commons Attribution 4.0 International License. The images or other third party material in this article are included in the article's Creative Commons license, unless indicated otherwise in the credit line; if the material is not included under the Creative Commons license, users will need to obtain permission from the license holder to reproduce the material. To view a copy of this license, visit http://creativecommons.org/licenses/by/4.0/

(C) The Author(s) 2016 